\title{
A Utilização de Softwares no Ensino e Aprendizagem dos Cursos de Engenharia
}

\author{
Software Use in the Teaching and Learning of Engineering Courses
}

\author{
Carlos Alberto Gonçalves da Silva Maistro Machado; ${ }^{\mathrm{a}}$ Angelica da Fontoura Garcia Silva*ab; Diego Fogaça Carvalho ${ }^{\mathrm{a}}$
}

\begin{abstract}
${ }^{a}$ Unopar, Programa de Pós-Graduação Stricto Sensu em Metodologias para o Ensino de Linguagens e suas Tecnologias. PR, Brasil.

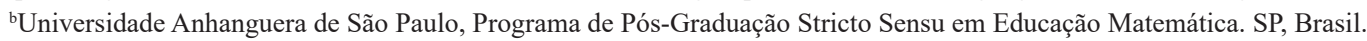

*E-mail: angelicafontoura@anhanguera.com
\end{abstract}

\begin{abstract}
Resumo
O estudo aqui exposto é parte de uma pesquisa de mestrado em andamento desenvolvida no Programa de Pós-Graduação Stricto Sensu em Metodologias para o Ensino de Linguagens e suas Tecnologias. Trata-se de uma investigação que tem o propósito de retratar a pesquisa acadêmica encontrada no repositório de publicações da Associação Brasileira de Ensino em Engenharia - ABENGE -. Para a realização desta pesquisa bibliográfica buscou-se no repositório. os artigos publicados em um período de 12 anos - a partir de 2006 até 2018 . Selecionou-se publicações que tiveram como foco a utilização de softwares para desenvolver processos de ensino e de aprendizagem de disciplinas de cursos de engenharia. Dos 397 artigos encontrados, 25 foram selecionados como instrumento de análise para esta pesquisa, dos quais, 14 deles foram publicados em 2017 e 2018. Analisando as tais publicações, pôde concluir que o método qualitativo se sobressaiu acima do quantitativo e quali-quanti, e ainda $44 \%$ dos artigos se basearam em questionários, avaliações ou entrevistas, seguidos de $40 \%$ de pesquisas bibliográficas. No tocante aos resultados foi possível identificar que quase a totalidade dos estudos concluiu que a utilização de diferentes metodologias, dentre elas softwares para o ensino, é fundamental para a inserção do futuro engenheiro ao mercado atual.
\end{abstract}

Palavras-chave: Ensino de Engenharia. Processos de Ensino e de Aprendizagem. Tecnologia Computacional.

\begin{abstract}
The study here presented is part of an ongoing master's research developed at the Stricto Sensu Postgraduate Program in Methodologies for Language Teaching and its Technologies. It is an investigation that has the purpose of portraying the academic research found in the repository of publications of the Brazilian Association of Engineering Teaching - ABENGE. In order to carry out this bibliographical research, the repository was searched for articles published over a period of 12 years - from 2006 to 2018. Publications that focused on the use of software to develop teaching and learning processes for engineering courses were selected. Of the 397 articles found, 25 were selected as analysis tools for this research, 14 of which were published in 2017 and 2018. Analyzing these publications, it was possible to conclude that the qualitative method stood out above the quantitative and quali-quantitative methods, and 44\% of the articles were based on questionnaires, evaluations or interviews, followed by $40 \%$ of bibliographical research. Regarding the results, it was possible to identify that almost all the studies concluded that the use of different methodologies, including software for teaching, is fundamental for the insertion of the future engineer in the current market.
\end{abstract}

Keyword: Engineering Education. Teaching and Learning Processes. Computational Technology.

\section{Introdução}

Com a inserção da tecnologia no nosso dia-a-dia, é impossível falar de educação e não abordar o seu uso, uma vez que todos estão imersos a ela. De acordo com Moran (2005), "as tecnologias, [...], estão trazendo fundamentalmente nestes últimos vinte anos a mobilidade total, a possibilidade de realizar atividades ou tarefas, sem necessariamente, ir a um lugar determinado. Contudo, a interatividade é uma alternativa para a realização das atividades citadas por Moran (2005), porém, é necessário haver a quebra de paradigmas do ensino tradicional e se render ao ensino com a inserção das Tecnologias de Informação e Comunicação (TIC). Um exemplo clássico do uso das TIC se dá por meio da utilização da metodologia de ensino denominada como sala de aula invertida, ou flipped class, em inglês, nela o aluno passa a ter o papel ativo dentro da sua aprendizagem, interagindo com atividades assíncronas antes da aula presencial. Considerando o exposto, acreditamos que as utilizações das tecnologias nas aulas de engenharia poderiam conferir diferentes resultados ao ensino-aprendizagem.

Sob o ponto de vista das políticas legais, é importante destacar que a Câmara de Educação Superior do Conselho Nacional de Educação (BRASIL, 2002), em seu artigo $5^{\circ}$ estabelece que o plano de ensino deve garantir a aprendizagem e perfil do formando. Assim, sabendo que cada vez mais o mercado exige conhecimentos tecnológicos, é emergente a compreensão de como e quando se utilizar de as TIC. Lembrando que segundo o artigo 53 da Lei de Diretrizes e Bases da Educação Nacional (BRASIL, 1996) as universidades possuem autonomia para alterar os currículos do curso, ou ainda, o plano pedagógico do 
curso (PPC) e, nesse contexto, consideramos que a pesquisa sobre a temática, torna-se relevante uma vez que a comunidade acadêmica pode levar em conta tais resultados quando elaborarem seus currículos e PPC.

Frente a isso, buscamos conhecer os trabalhos produzidos nos últimos 13 anos que abordem a questão exposta, assim, realizamos uma pesquisa sistemática dentro do portal da Associação Brasileira de Educação em Engenharia (ABENGE), uma vez que esta Associação busca disseminar o conhecimento comum à área de ensino de engenharia, promovendo conteúdos que possam contribuir para a evolução da área referida.

\section{Desenvolvimento}

\subsection{Metodologia}

Neste artigo apoiados em Lakatos e Marconi (2001) expomos resultados de uma pesquisa bibliográfica, tida como:

[...]bibliografia já tornada pública em relação ao tema estudado, desde publicações avulsas, boletins, jornais, revistas, livros, pesquisas, monografias, teses, materiais cartográficos, etc. [...] e sua finalidade é colocar o pesquisador em contato direto com tudo o que foi escrito, dito ou filmado sobre determinado assunto [...]. (LAKATOS E MARCONI, 2001, p. 183, grifos nossos).

Assim, para entrar "em contato direto com tudo o que foi escrito" no Congresso Brasileiro de Educação e Engenharia realizamos uma busca de artigos e resumos dentro do seu portal com a finalidade de conhecer o que vem sendo publicado na área do ensino da engenharia com a utilização de softwares nos últimos 13 anos, mais especificamente nas disciplinas que compõem a grade do curso superior de engenharia. Para isso, selecionamos e aplicamos duas palavras chaves no dispositivo de busca do portal: Software e Ensino-Aprendizagem, pesquisadas sem a utilização de aspas.

Para alcançar o número de 25 artigos, apresentados a seguir nos resultados, analisamos os resumos dos trabalhos encontrados, com o propósito de selecionar os que possuem características mais próximas à proposta aqui desejada.

\subsection{Análise e discussão}

Diferentes trabalhos foram encontrados, totalizando 397 publicações as quais foram encontradas em dois intervalos de tempo, conforme Quadro 1 exposto a seguir:

Quadro 1 - Quantidade de artigos publicados por intervalo

\begin{tabular}{|c|c|}
\hline Intervalo de Tempo - Anos & Quantidade de Artigos \\
\hline $2006-2014$ & 280 \\
\hline $2015-2018$ & 117 \\
\hline
\end{tabular}

Fonte: Dados da pesquisa. .

No primeiro intervalo - 2006 a 2014 - encontramos 280 publicações localizadas através da digitação de palavras chaves contidas dentro dos seus resumos e títulos, utilizando a ferramenta de busca oferecida pelo site. Já no segundo intervalo - 2015 a 2018 - foram encontradas 117 publicações por meio da leitura dos títulos, uma vez que o recurso de busca não estava oferecido no site. É importante salientar que no período da pesquisa, os repositórios dos anos de 2013 e 2015 não estavam disponíveis para consulta, assim, esta investigação não considerou as publicações destes anos.

A partir desta primeira seleção sistematizada - 397 estudos foram encontrados - contudo, fizemos uma segunda seleção, com o propósito de eliminar publicações que não se remetiam aos softwares no ensino e aprendizagem e/ou a engenharia. Analisamos minuciosamente todos os títulos encontrados, o que resultou em 50 trabalhos. Para identificar quais estudos realmente poderiam ser escopo deste estudo realizamos uma nova análise, desta vez focando no que estava descrito nos resumos a fim identificar a sincronicidade entre o objetivo dos trabalhos filtrados e este que se lê. Por meio desta análise, 25 foram selecionados, os quais estão apresentados no Quadro 2 exposto a seguir.

Quadro 2 - Resultado dos artigos pesquisados.

\begin{tabular}{|l|l|l|}
\hline \multicolumn{1}{|c|}{ AUTOR } & \multicolumn{1}{c|}{ Título } & Ano \\
\hline $\begin{array}{l}\text { RIOS; BACELAR; } \\
\text { CARVALHO }\end{array}$ & $\begin{array}{l}\text { Análise de metodologias de execução de projetos aplicadas à disciplina de instalações hidráulicas e } \\
\text { sanitárias prediais na universidade de fortaleza }\end{array}$ & 2018 \\
\hline SILVA et. al.. & Desenvolvimento de software educacional para aprimoramento do ensino da resistência dos materiais & 2018 \\
\hline MENDES et. al.. & $\begin{array}{l}\text { Análise do curso de engenharia civil da universidade federal de ouro preto de acordo com a percepção } \\
\text { dos alunos }\end{array}$ & 2018 \\
\hline BARROS et al.. & Sistemas mecânicos treliçados para auxiliar o aprendizado prático em mecânica geral & 2018 \\
\hline MELO; MOCOCK & $\begin{array}{l}\text { Experiências didático-pedagógicas na educação em engenharia: a importância dos programas de } \\
\text { monitoria e das tecnologias educacionais }\end{array}$ & 2018 \\
\hline SARAGOSA & $\begin{array}{l}\text { Capacitação docente e a personalização do processo de ensino-aprendizagem de engenharia estrutural } \\
\text { no contexto das tecnologias de informação e comunicação }\end{array}$ & 2018 \\
\hline SILVA et al.. & $\begin{array}{l}\text { Interação teoria e prática no ensino da engenharia: uma experiência pedagógica em cursos de } \\
\text { engenharia }\end{array}$ & 2018 \\
\hline $\begin{array}{l}\text { MASCARENHAS; } \\
\text { DIAS; BONALDO; } \\
\text { E.. }\end{array}$ & $\begin{array}{l}\text { Impactos da utilização dos softwares no processo de ensino-aprendizagem na disciplina de teoria de } \\
\text { estruturas na PUC minas barreiro }\end{array}$ & 2017 \\
\hline MENDES et. al.. & $\begin{array}{l}\text { Estudo preliminar para introdução de software algébrico computacional no ensino de cálculo } \\
\text { diferencial }\end{array}$ & 2017 \\
\hline
\end{tabular}




\begin{tabular}{|c|c|c|}
\hline AUTOR & Título & Ano \\
\hline SILVA; OLIVEIRA & $\begin{array}{l}\text { Softwares didáticos gratuitos e de código aberto: ferramentas para potencializar o ensino e } \\
\text { aprendizagem das engenharias }\end{array}$ & 2017 \\
\hline $\begin{array}{l}\text { FREITAS; } \\
\text { ALENCAR; } \\
\text { CAVALCANTE }\end{array}$ & $\begin{array}{l}\text { Alternativa metodológica no processo de ensino-aprendizagem em desenho técnico e em desenho } \\
\text { assistido por computador }\end{array}$ & 2017 \\
\hline PASSOS et. al.. & $\begin{array}{l}\text { Uso de softwares para auxílio educacional em cursos de engenharia civil no brasil: um mapeamento } \\
\text { sistemático }\end{array}$ & 2017 \\
\hline $\begin{array}{l}\text { CAVALCANTE } \\
\text { et. al.. }\end{array}$ & $\begin{array}{l}\text { Análise sobre o ensino utilizando ferramentas de simulação no curso de engenharia civil da } \\
\text { universidade federal de alagoas }\end{array}$ & 2017 \\
\hline $\begin{array}{l}\text { ZALUSKI; } \\
\text { DANTAS }\end{array}$ & $\begin{array}{l}\text { Aplicação de softwares de simulação na educação em engenharia: um relato de experiências } \\
\text { internacionais exitosas em cursos de modelagem e simulação de sistemas }\end{array}$ & 2017 \\
\hline AGUIAR et. al.. & Utilização de aplicativos para dispositivos móveis no ensino de engenharia civil & 2016 \\
\hline $\begin{array}{l}\text { BERNARDINIS; } \\
\text { BUHER }\end{array}$ & $\begin{array}{l}\text { Alternativas pedagógicas inovadoras aprimorando o processo de ensino-aprendizagem no curso de } \\
\text { engenharia civil da Universidade Federal do Paraná }\end{array}$ & 2016 \\
\hline GAGLIARDO et al. & Engenharia civil: um desafio de formação & 2016 \\
\hline $\begin{array}{l}\text { MASCARENHAS; } \\
\text { BONALDO; DIAS. }\end{array}$ & O uso do MATLAB no processo de ensino-aprendizagem nos cursos de engenharia civil & 2016 \\
\hline $\begin{array}{l}\text { ZAU; PACHECO; } \\
\text { BERNARDINIS }\end{array}$ & $\begin{array}{l}\text { Um estudo da correlação entre o estilo de ensino-aprendizagem dos alunos e docentes do curso de } \\
\text { engenharia civil da Universidade Federal do Paraná }\end{array}$ & 2016 \\
\hline $\begin{array}{l}\text { MAIAS; BORGES; } \\
\text { CALIXTO }\end{array}$ & Desenvolvimento da aprendizagem sobre estruturas de concreto & 2014 \\
\hline $\begin{array}{l}\text { RAMIRO; } \\
\text { ANDREATTA- } \\
\text { DA-COSTA; } \\
\text { BERNARDES } \\
\end{array}$ & $\begin{array}{l}\text { Softwares educacionais - seu uso e importância no ensino-aprendizagem dos alunos de Engenharia } \\
\text { Civil }\end{array}$ & 2014 \\
\hline ROCHA et. al.. & Metaldim: software educativo para dimensionamento de estruturas metálicas & 2014 \\
\hline PEREIRA et. al.. & $\begin{array}{l}\text { Professores engenheiros: processo de construção da prática pedagógica no curso de engenharia de } \\
\text { produção civil da UNEB }\end{array}$ & 2012 \\
\hline $\begin{array}{l}\text { CAMPOS NETO } \\
\text { et. al.. }\end{array}$ & $\begin{array}{l}\text { Estudo comparativo de ferramentas computacionais que utilizam tecnologia BIM para o } \\
\text { desenvolvimento de projetos de engenharia }\end{array}$ & 2012 \\
\hline RABELO et. al.. & A realidade do setor da construção civil e o desafio dos docentes das engenharias & 2006 \\
\hline
\end{tabular}

Fonte: Dados da pesquisa.

Realizada a análise dos trabalhos relacionados, tem-se um resultado bimodal para os dois últimos anos de 2017 e 2018 - sete estudos - os quais representaram também a maior parte dos artigos selecionados $-56 \%$ do total. Em contrapartida, somente três estudos foram apresentados nos anos de 2006 e $2012-12 \%$ dos artigos selecionados.

Inicialmente, procuramos analisar quais os títulos desses artigos selecionados - 25 - que, de maneira geral, traziam indícios de discutirem novos processos pedagógicos na engenharia, com a utilização de atividades voltadas para a prática pedagógica com tecnologias, resultando assim em 15 dos 25 artigos.

Em um segundo momento, realizamos uma análise dos trabalhos quanto aos seus objetivos, as suas metodologias, e por fim, as suas conclusões. Ao que tange os objetivos, quatro grandes grupos puderam ser elencados: Os trabalhos que possuem o objetivo de avaliar o desempenho dos seus alunos através de atividades avaliativas; os artigos que visam avaliar os cursos de engenharia e como os alunos têm sido formados perante às exigências do mercado atual; as publicações que almejaram apresentar metodologias alternativas de ensino, excluindo aquelas que utilizam softwares; e, por fim, os trabalhos que buscaram metodologias diferenciadas utilizando softwares, todos elencados conforme o Quadro 3.
Quadro 3 - Objetivos dos trabalhos analisados

\begin{tabular}{|l|c|}
\hline Objetivo - Avaliar, Apresentar & $\mathbf{N}^{\mathbf{0}}$ de Trabalhos \\
\hline O desempenho dos alunos & 2 \\
\hline O curso e a formação dos alunos & 4 \\
\hline Metodologias alternativas & 7 \\
\hline Metodologias com softwares & 12 \\
\hline
\end{tabular}

Fonte: Dados da pesquisa.

Em relação à metodologia aplicada pelos autores, foi possível identificar que os estudos, de maneira geral, foram caracterizados como qualitativos sobre uma visão avaliativa, como indicam as Figuras 1 e 2.

Figura 1 - Método investigativo

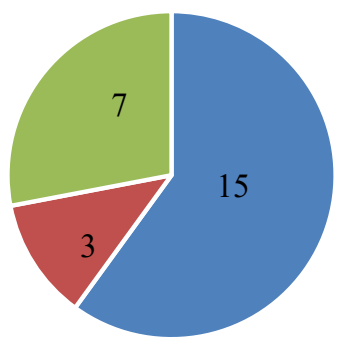

- Qualitativo $\quad$ Quantitativo $\backsim$ Quali-Quanti Fonte: Dados da pesquisa. 
Figura 2 - Método de levantamento dos dados

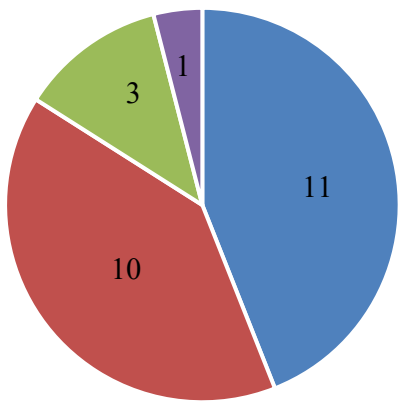

- Questionarios/Avaliações/Entrevistas

- Pesquisa Bibliográfica

- Observação da Prática

- Observação da Prática e Avaliação

Fonte: Dados da pesquisa.

Analisando a Figura 1, é possível identificar que o método qualitativo de investigação foi utilizado em $60 \%$ dos artigos selecionados, em contrapartida, $28 \%$ dos trabalhos foram investigados pelo método quali-quanti e apenas $12 \%$ pelo método quantitativo. Em relação ao levantamento dos dados, como pode ser visto na Figura 2, em geral, os trabalhos se basearam em Questionários (ou avaliações ou entrevistas) e Pesquisas Bibliográficas. Quanto a essa última categoria observamos que a maior parte dos trabalhos, se não todos, utilizam de Pesquisas Bibliográficas totalizando assim, 44\% e $40 \%$ respectivamente, sendo completados por observação de práticas, como a elaboração de uma ponte de palito de sorvete ou de macarrão, como publicaram Barros, et al. (2018) e Silva, et al. (2018), e pelo método experimental seguido de uma avaliação, obtendo respectivamente $12 \%$ e $04 \%$.

De maneira praticamente unanime - 96\% dos estudos - concluíram que as utilizações de novas metodologias são primordiais para a inserção do aluno no mercado atual, como citam Bernardinis e Buher (2016) "[...] que metodologias inovadoras utilizadas em sala de aula potencializaram o aprendizado da disciplina [...]", da mesma forma Locatelli (2009) também discute a importância do uso das tecnologias da informação tanto para o ensino superior como para a educação básica:

As TIC promovem, tanto na educação básica quanto no ensino superior, a leitura crítica e a produção de informação e comunicação como tema transversal dos conteúdos curriculares das diversas áreas do conhecimento. Isso constitui um novo paradigma para o processo de ensinoaprendizagem requerendo tanto do professor quanto dos alunos mudanças que não diminuirão o esforço disciplinado do estudo, mas podem potencializar o tempo e encurtar os espaços, articulando inúmeros indivíduos numa comunidade de aprendizagem (LOCATELLI, 2009, p.81).

Concordamos com o autor acerca das vantagens da TIC uma vez que possibilita aprimorar a disponibilidade de tempo e diminuir os espaços de forma a favorecer o desenvolvimento de trabalho em conjunto e ao mesmo tempo também acreditamos que para que isso ocorra é necessário que os atores envolvidos
- tanto professores como estudantes - tenham um novo olhar para os significados do ensinar e aprender nesse contexto. Tais observações estão em consonância com os pressupostos indicados no Art. $2^{\circ}$ da Lei de Diretrizes e Bases da Educação Nacional (LDBEN) de 1996, “A educação, [...] tem por finalidade o pleno desenvolvimento do educando, seu preparo para o exercício da cidadania e sua qualificação para o trabalho."

Concernente a avaliação de software encontramos Barros et al. (2018) avaliação positiva para a utilização do software FTool na aplicação prática do modelo no sistema "Mola Structural Kit" . Segundo os autores foi constatado que "[...] os alunos trabalharam com entusiasmo e motivação, desempenhando, em cada unidade, um papel de agente do processo de aprendizagem [...]", assim, deixando o método expositivo e passivo do ensino tradicional e passando ao ensino ativo, onde o aluno desenvolve o seu saber, auxiliando ainda o aluno à superar as suas dificuldades como trazem Melo e Mocock (2018).

Assim, para levar o aluno a construir o seu conhecimento, é importante que as metodologias de ensino sejam atualizadas proporcionando, por exemplo, a dinamicidade dos softwares como caracterizam Cavalcant et al. (2017). Além disso, é preciso levar em consideração resultados de pesquisas e documentos curriculares como por exemplo as Diretrizes Curriculares Nacionais, sem deixar a teoria de lado, procedimentos os quais estão totalmente atrelados à formação do engenheiro.

$\mathrm{Na}$ contramão da maior parte das conclusões analisadas, Rios, Bacelar e Carvalho (2018) por meio dos resultados de suas avaliações, constatam que os alunos que realizaram os cálculos e projeto à mão obtiveram resultados superiores aos alunos que utilizaram o software de desenho.

Embora a utilização dos softwares auxilie os alunos e os profissionais, possibilitando o engajamento entre o aluno e a disciplina (MAIA; BORGES; CALIXTO, 2014) é fundamental ter atenção às metodologias, ao público e suas avaliações.

De acordo com a LDBEN (1996) os cursos superiores devem incluir em seu ensino, os seguintes itens:

I - estimular a criação cultural e o desenvolvimento do espírito científico e do pensamento reflexivo;

II - formar diplomados nas diferentes áreas de conhecimento, aptos para a inserção em setores profissionais e para a participação no desenvolvimento da sociedade brasileira, e colaborar na sua formação contínua;

III - incentivar o trabalho de pesquisa e investigação científica, visando o desenvolvimento da ciência e da tecnologia e da criação e difusão da cultura, e, desse modo, desenvolver o entendimento do homem e do meio em que vive;

IV - promover a divulgação de conhecimentos culturais, científicos e técnicos que constituem patrimônio da humanidade e comunicar o saber através do ensino, de publicações ou de outras formas de comunicação;

$\mathrm{V}$ - suscitar o desejo permanente de aperfeiçoamento cultural e profissional e possibilitar a correspondente concretização, integrando os conhecimentos que vão sendo adquiridos numa 
estrutura intelectual sistematizadora do conhecimento de cada geração;

VI - estimular o conhecimento dos problemas do mundo presente, em particular os nacionais e regionais, prestar serviços especializados à comunidade e estabelecer com esta uma relação de reciprocidade;

VII - promover a extensão, aberta à participação da população, visando à difusão das conquistas e benefícios resultantes da criação cultural e da pesquisa científica e tecnológica geradas na instituição.

VIII - atuar em favor da universalização e do aprimoramento da educação básica, mediante a formação e a capacitação de profissionais, a realização de pesquisas pedagógicas e o desenvolvimento de atividades de extensão que aproximem os dois níveis escolares. (BRASIL, 1996)

Por fim, é importante validar o Art. 43 da LDBEN citado à cima. Já discutido ao longo deste instrumento, o docente tem papel fundamental uma vez que ele terá que guiar o aluno para com a sua aprendizagem e lhe preparar para o mercado de trabalho. Conforme as Diretrizes Nacionais (1996) devem haver a divulgação técnica por meio do ensino, de forma a estimular a compreensão dos problemas presentes no mundo, como por exemplo o estudo de casos reais em sala de aula, provocando no aluno a busca pelo aperfeiçoamento cultural, cientifico e profissional.

\section{Conclusão}

Como já citado, o mercado de trabalho vem exigindo cada vez mais do profissional, seja na interdisciplinaridade ou no domínio das TIC, as quais fazem parte do seu dia-a-dia. É notória a necessidade da atualização dos métodos pedagógicos aplicados pelos professores das mais diversas áreas, entretanto, na engenharia civil é ainda mais evidente, uma vez que é por meio da utilização de softwares que os alunos podem compreender, de forma mais rápida o comportamento das estruturas calculadas através dos exaustivos cálculos em folha de papel. Os softwares de maneira geral, vem para agregar no cotidiano do engenheiro, lhe dando maior praticidade e rapidez nos processos de cálculo. Entretanto, estudos analisados destacam que a deficiência existente na qualificação dos engenheiros, se dá pelo fato da falta de atualização do sistema de ensino perante as exigências do mercado. Ainda no contexto da utilização de novas metodologias, é importante frisar que as pesquisas mostram que o embasamento teórico ainda se faz necessário, podendo utilizar os softwares como complemento às aulas, como destacam.

Tomando os resultados deste instrumento, foi possível encontrar diversos trabalhos que abordam o ensino e aprendizagem na engenharia, aumentando gradativamente a quantidade de publicações a partir de 2006, o que demonstra, que existem outros pesquisadores preocupados com a necessidade da atualização da didática em engenharia. Neste âmbito, sugere-se que os cursos devem proporcionar ao aluno um ensino conforme o cenário do mercado atual, e ainda, estimular eles neste quesito.

Por fim, este instrumento tem como objetivo instigar o leitor a pensar exatamente nos aspectos aqui expostos, e o porquê de não aceitar a mudança do cenário do aprendizado e romper com os sistemas tradicionais, deixando a zona de conforto, uma vez que as TIDIC são um caminho sem volta e que acordo com o Art. 53 da LDBEN (1996) as universidades possuem autonomia para alterar suas metodologias.

\section{Referências}

AGUIAR, A.L.S.D. et al. Utilização de aplitavios para dispositivos móveis no ensino de Engenharia Civil. In: CONGRESSO BRASILEIRO DE EDUCAÇÃO EM ENGENHaria (COBENGE), 2016.

BARROS, P.D.M.A. et al. Sistemas mecânicos treliçados para auxiliar o aprendizado prático em mecânica geral. In: CONGRESSO BRASILEIRO DE EDUCAÇÃO EM ENGENHARIA; SIMPÓSIO INTERNACIONAL DE EDUCAÇÃO EM ENGENHARIA, Salvador,2018.

BERNARDINIS, M.A.P.; BUHER, B. M. C. Alternativas pedagógicas inovadoras aprimorando o processo de ensinoaprendizagem no curso de Engeharia Civil da Universidade Federal do Paraná. In: CONGRESSO BRASILEIRO DE EDUCAÇÃO EM ENGENHARIA, 2016.

BRASIL. Lei ${ }^{0}$ 9.394, de 20 de dezembro de 1996. Brasília: Diário Oficial da União, 1996. Disponivel em: <http://www. planalto.gov.br/ccivil_03/Leis/L9394.htm>. Acesso em: $23 \mathrm{dez}$. 2020.

BRASIL. Ministério da Educcação Institui Diretrizes Curriculares Nacionais do Curso de Graduação em Engenharia. Resolução CNE/CES 11 de 11 de março de 2002. Brasília: Diário Oficial da União, 2002. Disponivel em: <http://portal.mec.gov.br/cne/ arquivos/pdf/CES112002.pdf>. Acesso em: 24 abr. 2020.

CAVALCANTE, A.F. et al. Análise sobre o ensino utilizando ferramentas de simulação no curso de Engenharia Civil da Universidade Federal de Alagoas. In: CONGRESSO BRASILEIRO DE EDUCAÇÃO EM ENGENHARIA (COBENGE), Joinville, 2017.

ELLWEIN, S.A.F.; KFOURI, S.F. As possibilidades e contribuições do hipertexto no ensino e aprendizagem. Rev. Ensin. Educ. Ciênc. Hum., v.17, n.2, p.120-125, 2016. doi: https:// doi.org/10.17921/2447-8733.2016v17n2p120-125

FREITAS, B.B.; ALENCAR, C.M.S.D.; CAVALCANTE, A.P.D.H. Alternativa metodológica no processo de ensinoaprendizagem em desenho técnico e em desenho assistido por computador. In: CONGRESSO BRASILEIRO DE EDUCAÇÃO EM ENGENHARIA (COBENGE), Joinville, 2017.

GAGLIARDO, D.P. et al. Engenharia civil: um desafio de formação. In: CONGRESSO BRASILEIRO DE EDUCAÇÃO EM ENGENHARIA, 2016.

LAKATOS, E. M.; MARCONI, M. A. Fundamentos da metodologia científica. São Paulo: Atlas, 2001.

LOCATELLI, O.C. Tecnologia e implicaçãoes na educação. Rev. Pedag. Unochapecó, v.11, n.22, p.71-83, 2009.

MAIA, E.V.; BORGES, O.N.; CALIXTO, J.M.F. Desenvolvimento da aprendizagem sobre estruturas de concreto armado. In: CONGRESSO BRASILEIRO DE EDUCAÇÃO EM ENGENHARIA (COBENGE), Juiz de Fora, Setembro 2014.

MASCARENHAS, F.J.R.; BONALDO, E.; DIAS, V.C. O uso do MATLAB no processo de ensino-aprendizagem nos cursos de Engenharia Civil. In: XLIV Congresso Brasileiro de Educação em Engenharia (COBENGE), 2016. 
MASCARENHAS, F. J. R.; DIAS, V.C.; BONALDO, E. Impactos da utilização dos softwares no processo de ensino-aprendizagem na disciplina de teoria de estruturas na PUC Minas Barreiro. In: XLV Congresso Brasileiro de Educação em Engenharia (COBENGE), Joinville, Setembro 2017. 12.

MELO, F.G.; MOCOCK, J.F.B. Experiências didáticopedagórigas na educação em engenharia: A importância dos programas de monitorias e das tecnologias educacionais. In: CONGRESSO BRASILEIRO DE EDUCAÇÃO EM ENGENHARIA; SIMPÓSIO INTERNACIONAL DE EDUCAÇÃO EM ENGENHARIA, Salvador, 2018.

MENDES, E. et al. Estudo preliminar para introdução de software algébrico computacional no ensino de cálculo diferencial. In: CONGRESSO BRASILEIRO DE EDUCAÇÃO EM ENGENHARIA (COBENGE). Joinville 2017.

MENDES, V.F. et al. Análise do curso de engenharia civil da Universidade Federal de Ouro Preto de acordo com a percepção dos alunos. In: CONGRESSO BRASILEIRO DE EDUCAÇÃO EM ENGENHARIA; SIMPÓSIO INTERNACIONAL DE EDUCAÇÃO EM ENGENHARia, Salvador, 2018.

MORAN, J.M. Avaliação das mudanças que as tecnologias estão provando na educação pesencial e a distância. Educ. Cultura Contemp., v.2, n.4, p.89-108, 2005.

NETO, S.D.C. et al. Estudo comparativo de ferramentas computacionais que utilizam tecnologia BIM para desenvilvimento de projetos de Engenharia Civil. In: CONGRESSO BRASILEIRO DE EDUCAÇÃO EM ENGENHARIA (COBENGE), Belém, 2012.

PASSOS, D.D.S. et al. Uso de softwares para auxílio educacional em cursos de Engenharia Civil no Brasil: um mapeamento sistemático. In: CONGRESSO BRASILEIRO DE EDUCAÇÃO EM ENGENHARIA (COBENGE). Joinville, 2017.

PEREIRA, T.R.D.S. et al. Professores Engenheiros: processo de construção da prática pedagógica no curso de Engenharia de Produção Civil da UNEB. In: CONGRESSO BRASILEIRO DE EDUCAÇÃO EM ENGENHARIA (COBENGE). Belém, 2012.

RABELO, P.F.R.; ZAPLA, L.S.; AMORIM, S.R.L.D. A realidade do setor da construção civil e o desafio dos docentes das Engenharias. In: CONGRESSO BRASILEIRO DE EDUCAÇÃO EM ENGEnharia (COBENGE), 2006. 12.

RAMIRO, F.D.S.; ANDREATTA-DA-COSTA, L.;
BERNARDES, J.D.A. Softwares educacionais - Seu uso e importância no ensino-aprendizagem dos alunos de Engenharia Civil. In: CONGRESSO BRASILEIRO DE EDUCAÇÃO EM ENGENHARIA (COBENGE), Juiz de Fora, 2014.

RIOS, M.F.; BACELAR, F.A.; CARVALHO, T.M. X. B. Análise de metodologias de execução de projetos aplicadas à disciplina de instações hidráulicas e sanitárias prediais na Universidade de Fortaleza. In: CONGRESSO BRASILEIRO DE EDUCAÇÃO EM ENGENHARIA; SIMPÓSIO INTERNACIONAL DE EDUCAÇÃO EM ENGENHARIA, Salvador, 2018.

ROCHA, D.P.B.D. et al. METALDIM: Software educativo PARA DIMENSIONAMENTO DE ESTRUTURAS METÁLICAS. IN: CONGRESSO Brasileiro de Educação em Engenharia (COBENGE), Juiz de Fora, 2014.

SARAGOSA, G.C. Capacitação docente e a personalização do processo de ensino-aprendizagem de Egenharia Estrutural no contexto das tecnologias de informação e comunicação. In: CONGRESSO BRASILEIRO DE EDUCAÇÃO EM ENGENHARIA; SIMPÓSIO INTERNACIONAL DE EDUCAÇÃO EM ENGENHARIA, Salvador, 2018.

SILVA, J.R. et al. Intereação teoria e praática no ensino da Engenharia: Uma experiência pedagógica em cursos de Engenharia. CONGRESSO BRASILEIRO DE EDUCAÇÃO EM ENGENHARIA ; SIMPÓSIO INTERNACIONAL DE EDUCAÇÃO EM ENGENHARIA, Salvador, 2018.

SILVA, V.M.; OLIVEIRA, W.D.S. Softwares didáticos gratuitos e de código aberto: Ferramentas para potencializar o ensino e aprendizagem das Engenharias. In: CONGRESSO BRASILEIRO DE EDUCAÇÃO EM ENGENHARIA (COBENGE), Joinville, 2017.

YIN, R.K. Estudo de caso: planejamento e métodos. Porto Alegre: Bookman, 2010.

ZALUSKI, P.R.D.S.; DANTAS, M.J.P. Aplicação de softwares de simulação em Engenharia: Um relato de experiências internacionais exitosas em cursos de modelagem e simulação de sistemas. In: CONGRESSO BRASILEIRO DE EDUCAÇÃO EM ENGENHARIA (COBENGE), Belém, 2017.

ZAU, S.K.S.; PACHECO, E.; BERNARDINIS, M.D.A P. Um estudo da correlação entre o estilo de ensino-aprendizagem dos alunos e docentes do curso de Engenharia Civil da Universidade Federal do Paraná. In: CONGRESSO BRASILEIRO DE EDUCAÇÃO EM ENGENHARIA (COBENGE), 2016. 